\title{
Kanggan Granule Ameliorates Dexamethasone-Induced Immunosuppression in Mice
}

\author{
Yumiao Gan', Manxia Gu1 ${ }^{1}$, Dongling Liư ${ }^{1}$, Hongjing Zhou ${ }^{1}$, Chenye Zeng1, Tingting Yang1, \\ Hui Li ${ }^{1}$, Funeng Geng ${ }^{2}$, Junrong Du1* \\ ${ }^{1}$ Department of Pharmacology, West China School of Pharmacy, Key Laboratory of Drug Targeting and Drug Delivery \\ System, Sichuan University, Chengdu, China \\ ${ }^{2}$ Sichuan Engineering Research Center for Medicinal Animals, Chengdu, China \\ Email: *dujr_1@163.com
}

How to cite this paper: Gan, Y.M., Gu, M.X., Liu, D.L., Zhou, H.J., Zeng, C.Y., Yang, T.T., Li, H., Geng, F.N. and Du, J.R. (2019) Kanggan Granule Ameliorates Dexamethasone-Induced Immunosuppression in Mice. Journal of Biosciences and Medicines, 7, 80-91.

https://doi.org/10.4236/jbm.2019.73008

Received: January 7, 2019

Accepted: March 11, 2019

Published: March 14, 2019

Copyright $\odot 2019$ by author(s) and Scientific Research Publishing Inc. This work is licensed under the Creative Commons Attribution International License (CC BY 4.0).

http://creativecommons.org/licenses/by/4.0/

(c) (i) Open Access

\begin{abstract}
Objective: This study was to investigate the effect of the Chinese herbal compound Kanggan granule $(\mathrm{KG})$ on immune function in a mouse model of immunosuppression and its possible mechanism of action. Method: ICR mice were randomly divided into a normal control group (untreated non-immunosuppressed, Control), untreated immunosuppressed group (Model), positive control group (immunosuppressed and treated with $1.6 \mathrm{~g} / \mathrm{kg}$ astragalus granule $[A G]$ ), high-dose $K G$ group (immunosuppressed and treated with $24 \mathrm{~g} / \mathrm{kg}, \mathrm{KG}-24$ ), and low-dose KG group (immunosuppressed and treated with $6 \mathrm{~g} / \mathrm{kg}, \mathrm{KG}-6$ ). Each group received intragastric administration once daily for 7 days. Immunosuppression was induced by an intraperitoneal injection of dexamethasone $(25 \mathrm{mg} / \mathrm{kg}$ ) once daily beginning on day 1 for 3 days. To illuminate the mechanism of immunomodulatory, we studied the effects of KG on nonspecific immunity, humoral immunity and cellular immunity in mice respectively. Results: KG improved organ weights and improved the phagocytic ability of mononuclear macrophages in immunosuppressed mice $(p<0.01$ or $p<0.05)$. The proliferation of spleen lymphocytes and number of peripheral blood leukocytes were enhanced after KG treatment in immunosuppressed mice $(p<0.05)$. KG increased the $\mathrm{CD} 4^{+} / \mathrm{CD}^{+}$ ratio in immunosuppressed mice $(p<0.01)$ and increased serum IL-2 and IgG levels $(p<0.01)$. Conclusions: KG can improve immune function in immunosuppressed mice. Nonspecific immunity, humoral immunity, and cellular immunity were all enhanced.
\end{abstract}

\section{Keywords}

Kanggan Granule, Immunomodulatory, Nonspecific Immunity, 
Humoral Immunity, Cellular Immunity

\section{Introduction}

The immune system is indispensable for our body. It protects against the invasion of foreign pathogens through nonspecific immunity, humoral immunity, and cellular immunity [1]. Researchers have found that the immune system plays an important role in both the induction and treatment of disease, including cancer, acquired immunodeficiency syndrome, and inflammation [2] [3] [4]. Therefore, studying drugs that act on the immune system is necessary so that immune system-related diseases can be more effectively treated.

The clinical practice of traditional Chinese medicine (TCM) has a long history that has resulted in a considerable number of useful preparations, and TCM is still widely used today [5] [6]. Kanggan granule (KG) is a Chinese herbal compound that is included in the 2015 Chinese Pharmacopoeia for treating cold viruses. KG consists of Jinyinhua (Lonicerae japonicae Flos, the dry flower bud or early blossoms of Lonicera japonica Thunb, Caprifoliaceae), Mianmaguanzhong (Dryopteridis Crassirhizomatis Rhizoma, the rhizome of Dryopteris crassirhizoma Naki, Dryopteridaceae), and Chishao (Paeoniae Radix Rubra, the root of Paeonia lactiflora Pall or Paeonia veitchii Lynch, Ranunculaceae). Lonicerae japonicae Flos has been reported to have antioxidant and antiinflammatory properties [7]. Dryopteridis Crassirhizomatis Rhizoma has been reported to be useful for the treatment of influenza virus [8]. Previous studies showed that Paeoniae Radix Rubra protects the liver and has antioxidant activity [9] [10]. Although the effects of KG on some immune system-related diseases have been reported in clinical studies [11] [12], little preclinical experimental information about KG is available. Additionally, the immunoregulatory effect of $\mathrm{KG}$ has not been reported. In the present study, we investigated the effects of KG on immune function in immunosuppressed mice and evaluated the mechanism of action of KG to provide a basis for adding the new indication of KG in the immune system.

\section{Materials and Methods}

\subsection{Animals}

Specific-pathogen-free male ICR mice (weighing 20 - 22 g) were supplied by Chengdu Dossy Experimental Animals Co. Ltd. (Chengdu, Sichuan, China). All of the animals were raised in plastic cage at $25^{\circ} \mathrm{C} \pm 2{ }^{\circ} \mathrm{C}$ and had free access to food and water. The animals were raised under a $12 \mathrm{~h} / 12 \mathrm{~h}$ light/dark cycle. The animal studies were conducted in accordance with the Regulations of Experimental Animal Administration issued by the State Committee of Science and Technology of the People's Republic of China. All of the procedures were approved by the Animal Research Committee of West China School of Pharmacy. 


\subsection{Reagents}

Kanggan granule (Sichuan Gooddoctor-Panxi Pharmaceutical Co., Ltd., Xichanng, Sichuan, China; batch No. 160703), Astragalus granule (AG; Sichuan Baili Pharmaceutical Co., Ltd., Chengdu, Sichuan, China; batch No. 161248), dexamethasone sodium phosphate injection (Tianjin Kingyork Group Co., Ltd., Tianjin, China; batch No. 1610092), RPMI 1640 Medium (Thermo Fisher Scientific, Waltham, MA, USA), Concanavalin A (ConA; Sigma-Aldrich, St. Louis, MO, USA), lipopolysaccharide (LPS; Sigma-Aldrich, St. Louis, MO, USA), MTT (Amresco Co., Solon, Ohio, USA), the mouse interleukin-2 (IL-2) enzyme-linked immunosorbent assay (ELISA) kit (Shanghai Yaji Biotechnology Co., Ltd., Shanghai, China), the mouse immunoglobulin G (IgG) ELISA kit (Nanjing SenBeijia Biotechnology Co., Ltd., Nanjing, Jiangsu, China), anti-CD3 (fluorescein isothiocyanate [FITC]; Thermo Fisher Scientific Co., Waltham, MA, USA), anti-CD4 (P-phycoerythrin [PE]; Thermo Fisher Scientific Co., Waltham, MA, USA), and anti-CD8 (PE-cy5; Thermo Fisher Scientific Co., Waltham, MA, USA) were used.

\subsection{Animal Groups and Drug Treatment}

Based on the previous reports [13], ICR mice were randomly divided into a normal control group (untreated and non-immunosuppressed, Control), untreated immunosuppressed group (Model), positive control group (immunosuppressed and treated with $1.6 \mathrm{~g} / \mathrm{kg} \mathrm{AG}$ ), immunosuppressed and treated with high-dose KG group (24 g/kg, KG-24), and immunosuppressed and treated with low-dose KG group (6 g/kg, KG-6). All of the doses were converted from the human clinical dose. With the exception of the normal control group, each group was given dexamethasone sodium phosphate injection $(25 \mathrm{mg} / \mathrm{kg})$ intraperitoneally once daily from day 1 to day 3 of the experiment to induce immunosuppression. From day 1 to day 7 of the experiment, the AG group, KG-24 group, and KG-6 group were orally treated with the respective doses daily. During the experiment, the mice in each group received the same feeding condition, and morphological characteristics of the mice were observed.

\subsection{Mononuclear Macrophage Phagocytosis and Organ Weight}

Macrophage phagocytosis was determined using a previously described method with modifications [14]. Body weight was measured on days 1 and 8 . On day 8 , India ink $(0.05 \mathrm{ml} / 10 \mathrm{~g})$ was injected in the caudal vein, and then at $1 \mathrm{~min}(\mathrm{t} 1)$ and $5 \mathrm{~min}$ (t5), $20 \mu \mathrm{l}$ of peripheral blood was collected respectively from the orbital vein. Peripheral blood was immediately added to $2 \mathrm{ml}$ of $0.1 \% \mathrm{Na}_{2} \mathrm{CO}_{3}$, and the optical density (OD) was measured at a wavelength of $680 \mathrm{~nm}$ (Varioskan Flash, Thermo Fisher Scientific Co., Waltham, MA, USA). The clearance rate, $k$, of charcoal particles $(K)$ was calculated as the following: $K=\log (\mathrm{OD} 1 / \mathrm{OD} 5) /(\mathrm{t} 5$ - t1). The phagocytic index, $\alpha$, was calculated as the following: $\alpha=$ (body weight/[liver weight + spleen weight] $) \times K^{1 / 3}$. The liver, thymus, and spleen were 
then removed and weighed. The organ index was calculated as organ weight/body weight.

\subsection{Proliferative Capacity of Splenic Lymphocytes}

The detection was guided by previous reports [13]. After the mice were sacrificed, the spleen was removed to prepare the splenocyte suspension $\left(1 \times 10^{7}\right.$ cells $\left./ \mathrm{ml}\right)$ with RPMI 1640 Medium. The splenocyte suspension was cultured in a 96-well plate (50 $\mu$ lper well, 9 wells/mouse). Lipopolysaccharide (which stimulates B-cells; $10 \mu \mathrm{g} / \mathrm{ml}, 50 \mu \mathrm{l} /$ well, 3 wells/group) and ConA (which stimulates T-cells; 5 $\mu \mathrm{g} / \mathrm{ml}, 50 \mu \mathrm{l} /$ well, 3 wells/group) were added to the splenocyte suspension, which was then cultured in the 96 -well plate. The cells were cultivated at $37^{\circ} \mathrm{C}$ with $5 \%$ $\mathrm{CO}_{2}$ for $48 \mathrm{~h}$. MTT ( $\left.10 \mu \mathrm{l}\right)$ was added to each well of the 96-well plate before the end of culturing. Four hours later, the OD was measured at a wavelength of 490 nm (Varioskan Flash, Thermo Fisher Scientific Co., Waltham, MA, USA) after discarding liquor, and $150 \mu \mathrm{l}$ of dimethylsulfoxide (DMSO) was added. The stimulation index, SI, was calculated as the following: $\mathrm{SI}=\mathrm{OD}_{\text {experimental well }} / \mathrm{OD}_{\text {blank }}$ well•

\subsection{Serum IL-2 and IgG Levels}

Serum was obtained by centrifuging peripheral blood at $1500 \times \mathrm{g}$ for $15 \mathrm{~min}$ according to the instructions of the IL-2 and IgG ELISA kits. The OD was measured at a wavelength of $450 \mathrm{~nm}$ (Varioskan Flash, Thermo Fisher Scientific Co., Waltham, MA, USA), and the concentrations of IL-2 and IgG were measured.

\subsection{Changes in Subsets of T-Lymphocyte in Peripheral Blood}

Detection of T-lymphocyte subsets was performed as described previously with modifications [13]. Peripheral blood was collected from the orbital vein, and the serum was discarded after centrifugation at $500 \times \mathrm{g}$ for $5 \mathrm{~min}$. Red blood cells were then lysed using red blood cell lysis buffer. Cells were resuspended in PBS. The lymphocyte suspension that was mixed with anti-CD3 was marked with FITC. The lymphocyte suspension that was mixed with anti-CD4 was marked with PE. The lymphocyte suspension that was mixed with anti-CD8 was marked with PE-Cy5. Each of the suspension mixtures was then incubated for $40 \mathrm{~min}$ on ice in the dark. The proportion of T-lymphocyte subsets was analyzed by flow cytometry (Beckman Coulter, Kraemer Boulevard Brea, CA, USA).

\subsection{Number of Peripheral Blood Leukocytes}

Following the previous reports [15], peripheral blood $(20 \mu \mathrm{l})$ was collected from the orbital vein, and $0.38 \mathrm{ml}$ of leukocyte diluent $(2.0 \mathrm{ml}$ of glacial acetic acid and $1.0 \mathrm{ml}$ of $1 \%$ gentian violet plus distilled water until a $100 \mathrm{ml}$ volume was reached) was added and then mixed. A small amount of the leukocyte suspension was added to the blood count chamber. Observing and counting the number of peripheral blood leukocytes under the microscope. 


\subsection{Hematoxylin-Eosin Staining}

The mice were transcardially perfused with phosphate-buffered saline (PBS). The spleen and thymus were removed, fixed in $4 \%$ paraformaldehyde, and embedded in paraffin. Paraffin sections $(5 \mu \mathrm{m})$ were prepared for hematoxylin-eosin (HE) staining.

\subsection{Statistical Analysis}

Using SPSS17.0 statistical software for experimental data processing and data were expressed as mean \pm SEM. The difference of data between groups was examined by one-way analysis of variance, and $p<0.05$ as the difference was statistically significant.

\section{Results}

\subsection{Effects of KG on Shape Characteristics of Mice}

At the beginning of the experiment, the appetite, fluid consumption, activity, and appearance of the mice were normal. A few days after KG administration, the appetite, fluid consumption, and appearance of the mice did not apparently change, but activity decreased compared with the normal control group. Immunosuppressed mice typically drink less, exhibit a decrease in appetite, and have low activity.

\subsection{Effects of KG on Nonspecific Immune Function in Immunosuppressed Mice}

The body weights of the mice in each group were generally the same before administration. Seven days after KG administration, body weight, the spleen index, the thymus index, the $K$-value, and the $\alpha$-value of the immunosuppressed mice significantly decreased compared with the normal control group $(p<0.01)$. The spleen index, $K$-value, and $\alpha$-value in the AG group, KG-24 group, and KG-6 group were higher compared with the untreated immunosuppressed group ( $p<$ 0.01 or $p<0.05$ ). The thymus index in the AG group, KG-24 group, and KG-6 group were not significantly different from the untreated immunosuppressed group ( $p>0.05$; Table 1$)$.

Hematoxylin-eosin staining revealed changes in the morphology of the spleen and thymus. Compared with the normal control group, the boundary of white pulp and red pulp was unclear in the untreated immunosuppressed group. The area of white pulp was lower in the untreated immunosuppressed group. More apoptotic cells appeared, and macrophages were rare in white pulp in the untreated immunosuppressed group. After treatment with AG and KG, the boundary between white and red pulp was clear. Fewer apoptotic cells were observed, and the number of macrophages increased in white pulp (Figure 1(A)). As shown in Figure 1(B), the thymus cortex became thinner in untreated immunosuppressed mice compared with the normal control group. The number of cells decreased in the thymus cortex in untreated immunosuppressed mice. The 
boundary between the thymus cortex and medulla was vague in the untreated immunosuppressed group. No differences in the morphology of the thymus were found between the AG and KG groups and the untreated immunosuppressed group.
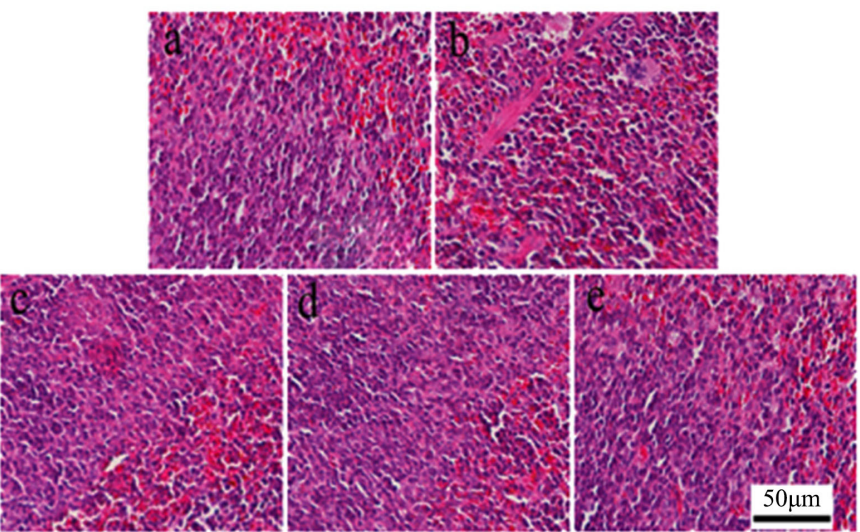

(A)

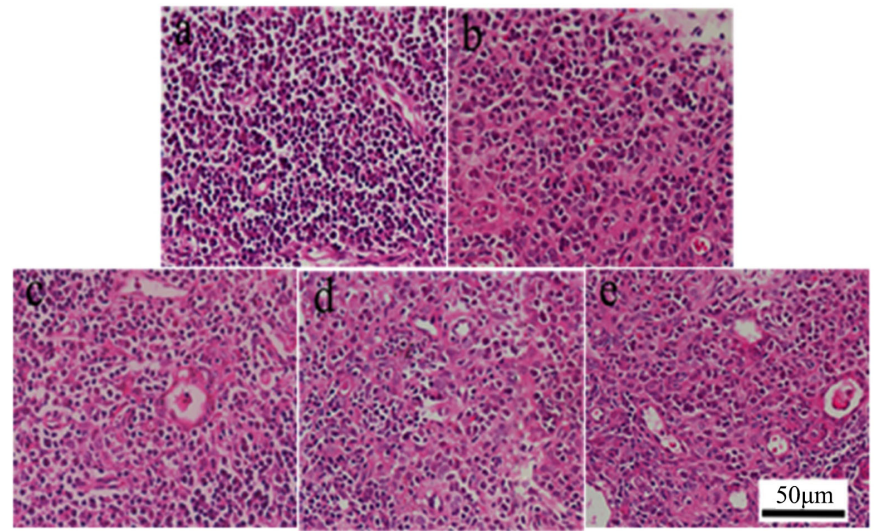

(B)

Figure 1. Effect of KG on the histomorphology of immune organs in immunosuppressed mice. (A) Hematoxylin-eosin staining of the spleen. (B) Hematoxylin-eosin staining of the thymus. (a) Normal control group. (b) Untreated immunosuppressed group. (c) Positive control (AG) group. (d) High-dose KG group. (e) Low-dose KG group.

Table 1. Effects of KG on mice organ index and the phagocytic ability of mononuclear macrophage (mean \pm SEM, $n=10$ ).

\begin{tabular}{|c|c|c|c|c|c|c|c|}
\hline \multirow{2}{*}{ Groups } & \multirow{2}{*}{$\begin{array}{c}\text { Dosage } \\
(\mathrm{g} / \mathrm{kg})\end{array}$} & \multicolumn{2}{|c|}{ Bodyweight (g) } & \multicolumn{2}{|c|}{ Organ index (mg/g) } & \multirow{2}{*}{$\mathrm{K}$} & \multirow{2}{*}{$\alpha$} \\
\hline & & Day 1 & Day 8 & Spleen & Thymus & & \\
\hline Control & - & $20.78 \pm 0.80$ & $25.13 \pm 1.25$ & $3.826 \pm 0.514$ & $2.357 \pm 0.325$ & $0.056 \pm 0.014$ & $7.357 \pm 0.875$ \\
\hline Model & - & $20.43 \pm 0.53$ & $23.18 \pm 0.82^{\# \#}$ & $2.527 \pm 0.610^{\# \#}$ & $0.785 \pm 0.158^{\# \#}$ & $0.037 \pm 0.010^{\# \#}$ & $5.831 \pm 0.829^{\# \#}$ \\
\hline AG & 1.6 & $20.57 \pm 0.51$ & $24.68 \pm 1.21^{\star \star}$ & $3.507 \pm 0.842^{\star *}$ & $0.800 \pm 0.220$ & $0.050 \pm 0.014^{*}$ & $6.832 \pm 0.790^{\star}$ \\
\hline KG-24 & 24 & $20.60 \pm 0.83$ & $24.28 \pm 0.94^{\star}$ & $3.300 \pm 0.973^{*}$ & $0.793 \pm 0.287$ & $0.048 \pm 0.010^{*}$ & $6.637 \pm 0.522^{\star}$ \\
\hline KG-6 & 6 & $20.77 \pm 1.34$ & $24.48 \pm 1.00^{*}$ & $3.357 \pm 0.723^{*}$ & $0.806 \pm 0.085$ & $0.050 \pm 0.008^{* *}$ & $7.076 \pm 0.462^{\star *}$ \\
\hline
\end{tabular}

${ }^{*} p<0.05,{ }^{* *} p<0.01$ vs. Model group; ${ }^{* *} p<0.01$ vs. Control group. 


\subsection{Effects of KG on Cellular Immune Function in Immunosuppressed Mice}

Twenty-four hours after the last administration, the proliferative capacity of splenic lymphocytes was investigated in vitro to determine the effect of KG. The SI of splenic lymphocytes that were stimulated by ConA in the untreated immunosuppressed group significantly decreased compared with the normal control group $(p<0.05)$. The SI of splenic lymphocytes in the AG group and KG-6 group was higher than in the untreated immunosuppressed group $(p<0.05)$. The SI in the KG-24 group was not significantly different from the untreated immunosuppressed group ( $p>0.05$; Figure 2(A)).

Serum IL-2 levels in the untreated immunosuppressed group significantly decreased compared with the normal control group $(p<0.01)$. Serum IL-2 levels in the AG group and KG-6 group were higher than in the untreated immunosuppressed group $(p<0.01)$. Serum IL-2 levels in the KG-24 group were not significantly different from the untreated immunosuppressed group $(p>0.05$; Figure 2(B)).

The $\mathrm{CD} 4^{+} / \mathrm{CD}^{+}$ratio in the untreated immunosuppressed group significantly decreased compared with the normal control group $(p<0.01)$. The $\mathrm{CD} 4^{+} / \mathrm{CD} 8^{+}$ ratio in the AG group, KG-24 group, and KG-6 group was higher than in the untreated immunosuppressed group $(p<0.01$; Figure $2(C)$ ).

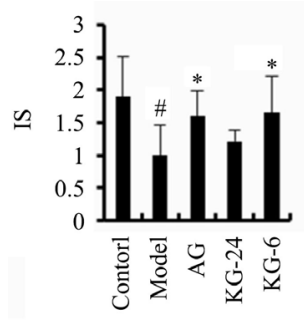

(A)

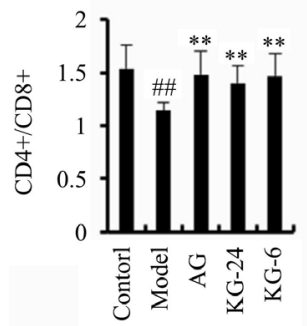

(C)

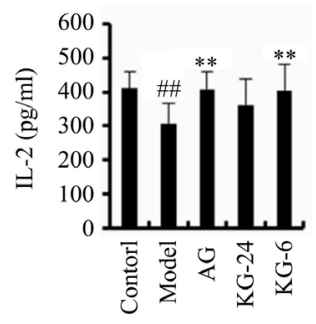

(B)

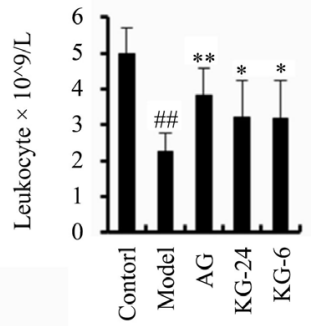

(D)

Figure 2. Effects of KG on cellular immune function in immunosuppressed mice. (A) The proliferative capacity of T-lymphocytes was stimulated by ConA $(5 \mu \mathrm{g} / \mathrm{ml})$, and the OD was measured at a wavelength of $490 \mathrm{~nm}$. $n=6$. (B) KG-6 increased serum IL-2 levels in immunosuppressed mice. $n=10$. (C) Flow cytometry analysis showed that KG-6 and KG-24 significantly increased the $\mathrm{CD}^{+} / \mathrm{CD}^{+}$ratio in immunosuppressed mice. $n=12$. (D) KG-6 and KG-24 significantly increased the number of leukocytes in immunosuppressed mice. $n=12$. Control, normal control group; Model, untreated immunosuppressed group. AG, positive control group; KG-24, high-dose KG group; KG-6, low-dose KG group. ${ }^{\star} p<0.05,{ }^{* *} p<0.01$, vs. Model group; ${ }^{\#} p<0.05,{ }^{\# \#} p<0.01$, vs. Control group. 
The number of peripheral blood leukocytes in the untreated immunosuppressed group significantly decreased compared with the normal control group $(p<0.01)$. The number of leukocytes in the AG group, KG-24 group, and KG-6 group was higher than in the untreated immunosuppressed group $(p<0.01$ or $p$ $<0.05$; Figure 2(D)).

\subsection{Effects of KG on Humoral Immune Function in Immunosuppressed Mice}

The SI of splenic lymphocytes that were stimulated by LPS in the untreated immunosuppressed group significantly decreased compared with the normal control group $(p<0.01)$. The SI of splenic lymphocytes in the AG group and KG-6 group was higher than in the untreated immunosuppressed group $(p<0.05)$. The SI in the KG-24 group was not significantly different from the untreated immunosuppressed group ( $p>0.05$; Figure 3(A)).

Serum IgG levels in the untreated immunosuppressed group significantly decreased compared with the normal control group $(p<0.01)$. Serum IgG levels in the AG group and KG-6 group were higher than in the untreated immunosuppressed group $(p<0.01)$. Serum IgG levels in the KG-24 group were not significantly different from the untreated immunosuppressed group $(p>0.05$, Figure $3(B))$.

\section{Discussion}

KG is often used to treat the cold virus. The overall course of a cold mainly consists of a conflict between the immune system and foreign pathogens. Another Chinese herbal compound, AG, is widely used today. Its main component is astragalus [Astragalus membranaceus (Fisch.) Bung., the dry root of Astragalus membranaceus (Fisch.) Bge. Var. mongholicus (Bge.) Hsiao or Astragalus membranaceus (Fisch.) Bge]. AG is also included in the 2015 Chinese Pharmacopoeia. The immunomodulatory effect of $A G$ has been reported [16]. The present study investigated the effects of KG on nonspecific immunity, humoral immunity, and cellular immunity in mice to evaluate the effect of KG and its possible mechanism of action.

Dexamethasone, a glucocorticoid, is widely used as immunosuppressive agent. In addition, dexamethasone is often used to induce immunosuppression as disease models in experimental studies [17] [18]. We developed a mouse model of dexamethasone-induced immunosuppression. Dexamethasone reduced organ weights of the mice and decreased the phagocytic ability of mononuclear macrophages. AG increased organ weights and increased the phagocytic ability of mononuclear macrophages in immunosuppressed mice. KG exerted similar effects, suggesting that it can regulate the nonspecific immune response.

Leukocytes are blood cells that play a primary role in the defense against foreign pathogens. Changes in the number of leukocytes are associated with some diseases [19] [20]. The present results showed that the number of leukocytes significantly decreased after dexamethasone administration, and the number of 


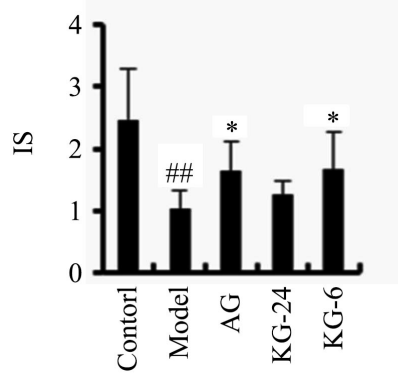

(A)

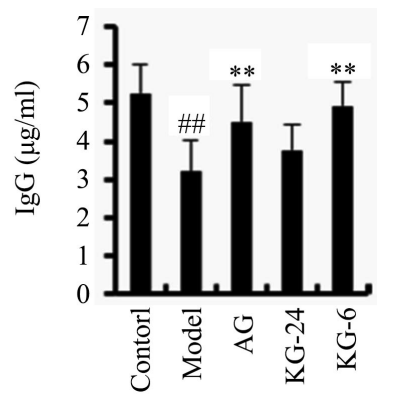

(B)

Figure 3. Effects of KG on humoral immune function in immunosuppressed mice. (A) The proliferative capacity of B-lymphocytes was stimulated by LPS $(10 \mu \mathrm{g} / \mathrm{ml})$, and the OD was measured at a wavelength of $490 \mathrm{~nm} . n=6$. (B) KG-6 increased serum IgG levels in immunosuppressed mice. $n=10$. Control, normal control group; Model, untreated immunosuppressed group; AG, positive control group; KG-24, high-dose KG group; KG-6, low-dose KG group. ${ }^{\star} p$ $<0.05,{ }^{* *} p<0.01$, vs. Model group; ${ }^{\# \#} p<0.01$, vs. Control group.

leukocytes significantly increased after KG and AG administration, indicating that KG can regulate the number of leukocytes in immunosuppressed mice. T-lymphocytes play an important role in cellular immunity. They comprise a large and complex group that can be divided into several subsets. In these subsets, $\mathrm{CD} 4^{+}$cells are known as helper T-cells, and $\mathrm{CD} 8^{+}$cells are known as cytotoxic T-cells. The $\mathrm{CD} 4^{+} / \mathrm{CD}^{+}$ratio can change when immunological diseases occur [21]. Therefore, the $\mathrm{CD} 4^{+} / \mathrm{CD}^{+}$ratio can be used to reflect the immune status of the body. In the present study, we investigated the effect of KG on the $\mathrm{CD} 4^{+} / \mathrm{CD}^{+}$ratio in peripheral blood in mice. The results showed that the $\mathrm{CD} 4^{+} / \mathrm{CD}^{+}$ratio in immunosuppressed mice was increased by $\mathrm{KG}$ and $\mathrm{AG}$ treatment, indicating that $\mathrm{KG}$ has a regulatory effect on cellular immunity.

The spleen contains a large number of lymphocytes, including B-lymphocytes and T-lymphocytes, which play an important role in humoral and cellular immunity. We investigated the proliferative capacity of B- and T-lymphocytes that were stimulated by LPS and ConA to evaluate changes in the immune response [22]. The results showed that KG effectively enhanced the proliferative capacity of splenic lymphocytes in immunosuppressed mice and regulated the humoral and cellular immune response.

IL-2 is produced by T-cells. It plays an important role in the immune system, including receptor activation, cytokine expression, and lymphocyte proliferation. Low levels of IL-2 reduce the immune response [23]. IgG is an antibody that plays a protective role in humoral immunity. When a body is invaded by foreign pathogens, serum IgG levels increase [24]. The levels of IL-2 and IgG can be measured to reflect the immune status of the body. The present results showed that serum IL-2 and IgG levels were significantly reduced in immunosuppressed mice. Both KG and AG increased serum IL-2 and IgG levels in immunosuppressed mice.

The results of KG-24 administration were not as promising as those of KG-6. 
The dose of KG in the the KG-24 group was likely much higher than the dose at which the drug reaches its maximal efficacy, whereas the dose of KG in the KG-6 group was likely slightly lower than the maximally effective dose. However, a more precise KG dose-response curve needs to be generated. To study the effect of KG on the immune system in mice, we developed a model of dexamethasone-induced immunosuppression. We then measured the phagocytic ability of mononuclear macrophages, organ weights, and the proliferative capacity of splenic lymphocytes, the number of peripheral blood leukocytes, the distribution of T-lymphocyte subsets, and serum IgG and IL-2 levels. The results of these analyses indicated that KG improved immunity and regulated immune function in immunosuppressed mice.

\section{Conclusion}

The present study showed that KG, which consists of Lonicerae japonicae Flos, Dryopteridis Crassirhizomatis Rhizoma, and Paeoniae Radix Rubra, can improve immune function in immunosuppressed mice, reflected by enhancements of nonspecific immunity, humoral immunity, and cellular immunity.

\section{Acknowledgements}

This work was supported in part by Targeting drug delivery system of Sichuan Province Youth Science and technology innovation team (2016TD0001).

\section{Conflicts of Interest}

The authors declare no conflicts of interest regarding the publication of this paper.

\section{References}

[1] Rios, J.L. (2010) Effects of Triterpenes on the Immune System. Journal of Ethnopharmacology, 128, 1-14. https://doi.org/10.1016/j.jep.2009.12.045

[2] Benton, T., Lynch, K., Dube, B., Gettes, D.R., Tustin, N.B., Ping Lai, J., Metzger, D.S., Blume, J., Douglas, S.D. and Evans, D.L. (2010) Selective Serotonin Reuptake Inhibitor Suppression of HIV Infectivity and Replication. Psychosomatic Medicine, 72, 925-932. https://doi.org/10.1097/PSY.0b013e3181f883ce

[3] Jellema, R., Passos, V., Zwanenburg, A., et al. (2013) Cerebral Inflammation and Mobilization of the Peripheral Immune System Following Global Hypoxia-Ischemia in Preterm Sheep. Journal of Neuroinflammation, 10, 807. https://doi.org/10.1186/1742-2094-10-13

[4] Liu, Y.N. and Zeng, G. (2012) Cancer and Innate Immune System Interactions: Translational Potentials for Cancer Immunotherapy. Journal of immunotherapy, 35, 299-308. https://doi.org/10.1097/CJI.0b013e3182518e83

[5] Chao, J., Dai, Y., Verpoorte, R., et al. (2017) Major Achievements of Evidence-Based Traditional Chinese Medicine in Treating Major Diseases. Biochemical pharmacology, 139, 94-104. https://doi.org/10.1016/j.bcp.2017.06.123

[6] Tsai, Y.T., Lai, J.N., Lo, P.C., Chen, C.N. and Lin, J.G. (2017) Prescription of Chinese Herbal Products Is Associated with a Decreased Risk of Invasive Breast Cancer. 
Medicine, 96, e7918. https://doi.org/10.1097/MD.0000000000007918

[7] Hsu, H.F., Hsiao, P.C., Kuo, T.C., et al. (2016) Antioxidant and Anti-Inflammatory Activities of Lonicera japonica Thunb. var. sempervillosa Hayata Flower Bud Extracts Prepared by Water, Ethanol and Supercritical Fluid Extraction Techniques. Industrial Crops and Products, 89, 543-549.

https://doi.org/10.1016/j.indcrop.2016.05.010

[8] Ou, C.B., Zhang, Q., Wu, G.J., Shi, N.N. and He, C. (2015) Dryocrassin ABBA, a Novel Active Substance for Use against Amantadine-Resistant H5N1 Avian Influenza Virus. Frontiers in microbiology, 6, 592.

https://doi.org/10.3389/fmicb.2015.00592

[9] Lee, S., Kwon, Y., Son, K., Kim, H. and Heo, M. (2005) Antioxidative Constituents from Paeonia lactiflora. Archives of Pharmacal Research, 28, 775-783. https://doi.org/10.1007/BF02977342

[10] Li, R.D., Guo, W.Y., Fu, Z.R., Ding, G.H., Zou, Y. and Wang, Z.X. (2011) Hepatoprotective Action of Radix Paeoniae Rubra Aqueous Extract against $\mathrm{CCl}_{4}$-Induced Hepatic Damage. Molecules, 16, 8684-8694. https://doi.org/10.3390/molecules16108684

[11] Yang, X.R. (2012) Clinical Observation on Treatment of 40 Cases of Upper Respiratory Tract Infection in Children. Guide of China Medicine, 10, 283-284.

[12] Ma, Z.P. and Li, G.G. (2011) The Efficacy of Kanggan Granule in Treating 36 Children with Hand-Foot-and-Mouth Disease. Chinese Journal of Experimental Traditonal Medical Formulae, 17, 269-270.

[13] Li, Y.H., Zheng, B., Tian, H.J., et al. (2017) Yupingfeng Powder Relieves the Immune Suppression Induced by Dexamethasone in Mice. Journal of Ethnopharmacology, 200, 117-123. https://doi.org/10.1016/j.jep.2017.01.054

[14] Cheng, X.B., Wang, C.L., Su, Y.Q., et al. (2018) Enhanced Opsonization-Independent phagocytosis and Highresponse Ability to Opsonized Antigen-Antibody Complexes: A New Role of Kupffer Cells in the Accelerated Blood Clearancephenomenon upon Repeated Injection of PEGylated Emulsions. Molecular Pharmaceutics, 15, 3755-3766. https://doi.org/10.1021/acs.molpharmaceut.8b00019

[15] Xu, S.Y. (2002) Experimental Methodology of Pharmacology. People's Medical Publishing House, Beijing.

[16] Xu, P.F., Sun, J.H., Gao, F.Y. and Liu, G.L. (2007) Experimental Study of Astragalus Granule on Immune Function of Mice. Chinese Journal of Information on Traditional Chinese Medicine, 14, 27-28.

[17] Johansen, P., Weiss, A., Bunter, A., Waeckerle-Men, Y., Fettelschoss, A., Odermatt, B. and Kundig, T.M. (2011) Clemastine Causes Immune Suppression through Inhibition of Extracellular Signal-Regulated Kinase-Dependent Proinflammatory Cytokines. The Journal of Allergy and Clinical Immunology, 128, 1286-1294. https://doi.org/10.1016/j.jaci.2011.06.023

[18] Sierra-Honigmann, M.R. and Murphy, P.A. (1992) T Cell Receptor-Independent Immunosuppression Induced by Dexamethasone in Murine $\mathrm{T}$ Helper Cells. The Journal of Clinical Investigation, 89, 556-560. https://doi.org/10.1172/JCI115620

[19] de Almeida, D.A., Rosa, S.I., da Cruz, T.C., et al. (2017) Mandevilla Longiflora (Desf.) Pichon Improves Airway Inflammation in a Murine Model of Allergic Asthma. Journal of Ethnopharmacology, 200, 51-59. https://doi.org/10.1016/j.jep.2017.02.015

[20] Maes, M., Lambrechts, J., Bosmans, E., et al. (1992) Evidence for a Systemic Immune Activation during Depression: Results of Leukocyte Enumeration by Flow 
Cytometry in Conjunction with Monoclonal Antibody Staining. Psychological Medicine, 22, 45-53. https://doi.org/10.1017/S0033291700032712

[21] Liu, J., Wang, L., Feng, Z., Geng, D., Sun, Y. and Yuan, G. (2017) Dynamic Changes of Laboratory Parameters and Peripheral Blood Lymphocyte Subsets in Severe Fever with Thrombocytopenia Syndrome Patients. International Journal of Infectious Diseases, 58, 45-51. https://doi.org/10.1016/j.ijid.2017.02.017

[22] Zhu, Z.Y., Meng, M., Sun, H., Li, Y., Ren, Y.Y. and Zhang, Y. (2016) Immunostimulatory Activity of Glycopeptides from Paecilomyces sinensis under Normal and Cyclophosphamide Induced Immunosuppressive Conditions in Mice Models. Food \& Function, 7, 3566-3576. https://doi.org/10.1039/C6FO00667A

[23] Jarosz, L., Kwiecien, M., Marek, A., Gradzki, Z., Winiarska-Mieczan, A., Kalinowski, M. and Laskowska, E. (2016) Effects of Feed Supplementation with Glycine Chelate and Iron Sulfate on Selected Parameters of Cell-Mediated Immune Response in Broiler Chickens. Research in Veterinary Science, 107, 68-74.

https://doi.org/10.1016/j.rvsc.2016.04.003

[24] Matos, L., Munoz, M.D.C., Molina, J.M., et al. (2017) Humoral Immune Responses of Experimentally Eimeria Ninakholyakimovae-Infected Goat Kids. Comparative Immunology, Microbiology and Infectious Diseases, 51, 60-65.

https://doi.org/10.1016/j.cimid.2017.04.002 